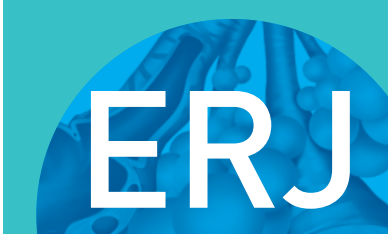

open research

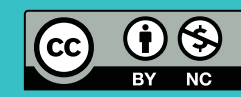

\title{
COPD: time to improve its taxonomy?
}

\author{
Bartolomé R. Celli ${ }^{1}$ and Alvar Agustín $2,3,4$ \\ Number 2 in the series "Gaps in our understanding of COPD" \\ Edited by A. Agustí and B. Celli
}

Affiliations: ${ }^{1}$ Brigham and Women's Hospital, Harvard Medical School, Boston, MA, USA. ${ }^{2}$ Respiratory Institute, Hospital Clinic, Universitat de Barcelona, Barcelona, Spain. ${ }^{3}$ Institut d'Investigacions Biomediques August Pi i Sunyer (IDIBAPS), Barcelona, Spain. ${ }^{4}$ CIBER Enfermedades Respiratorias, Spain.

Correspondence: Bartolomé R. Celli, Pulmonary and Critical Care Division, Brigham and Women's Hospital, 75 Francis Street, Boston, MA 02115, USA. E-mail: bcelliacopdnet.org

ABSTRACT Due to well-conducted epidemiological studies and advances in genetics, molecular biology, translational research, the advent of computed tomography of the lungs and bioinformatics, the diagnosis of chronic obstructive pulmonary disease (COPD) as a single entity caused by susceptibility to cigarette smoke is no longer tenable. Furthermore, the once-accepted concept that COPD results from a rapid and progressive loss of lung function over time is not true for a sizeable proportion of adults with the disease. Now we know that some genetic predisposition and/or different environmental interactions (nutritional, infectious, pollution and immunological) may negatively modulate post-natal lung development and lead to poorly reversible airflow limitation later in life, consistent with COPD. We believe it is time to rethink the taxonomy of this disease based on the evidence at hand. To do so, we have followed the principles outlined in the 1980s by J.D. Scadding who proposed that diseases can be defined by four key characteristics: 1) clinical description (syndrome), 2) disorder of structure (morbid anatomy), 3) disorder of function (pathophysiology) and 4) causation (aetiology). Here, we propose a pragmatic approach to the taxonomy of COPD based on different processes that result in a similar syndromic presentation. It can accommodate changes over time, as the pathobiology that may lead to COPD expands. We hope that stakeholders in the field may find it useful to better define the patients now boxed into one single entity, so that specific studies can be designed and conducted for each type of COPDs.

@ERSpublications

The ERJ Open Research series on gaps in our understanding of COPD tackles the subject of taxonomy http://ow.ly/TOYn30hlIA1

Cite this article as: Celli BR, Agustí A. COPD: time to improve its taxonomy? ERJ Open Res 2018; 4: 00132-2017 [https://doi.org/10.1183/23120541.00132-2017].

Previous articles in this series: No. 1: Agustí A, Celli BR. Natural history of COPD: gaps and opportunities. ERJ Open Res 2017; 3: 00117-2017.

Received: Oct 272017 | Accepted: Nov 162017

Conflict of interest: Disclosures can be found alongside this article at openres.ersjournals.com. Both authors are members of the Board of Directors of the Global Initiative for Chronic Obstructive Lung Disease (GOLD), but the opinions and proposals presented in this manuscript are their own and not necessarily those of GOLD.

Copyright $\odot$ ERS 2018. This article is open access and distributed under the terms of the Creative Commons Attribution Non-Commercial Licence 4.0. 


\section{Introduction}

Chronic obstructive pulmonary disease (COPD) is receiving increased attention because it has risen as a cause of morbidity and mortality in the world. Currently, it is the third most important noncommunicable disease, accounting for $5.3 \%$ of all deaths worldwide [1]. Paralleling its increased importance, the output of literature addressing the genetics, pathophysiology, natural history, clinical manifestations and therapeutic approaches of COPD have multiplied, reaching a total of over 15000 citations in PubMed for 2016 alone. Many of the old concepts have been revised and central to this is the general acceptance that COPD is not just one single entity, but rather a complex and heterogeneous one that may result from different causative agents that run different clinical courses and may benefit from some common therapies, but may require different individual approaches to improve outcomes $[2,3]$. Thus, current knowledge points to COPD to be more of a syndrome than a single disease.

This review argues that our current understanding of the causes resulting in "COPD" have to be somehow incorporated into its definition and taxonomy if we are to achieve the goal of implementing personalised therapy for these patients [4]. We do not address the controversial subject of the specific spirometric threshold needed to diagnose pathological airflow limitation because it does not influence the taxonomic definitions proposed.

\section{COPD: disease or syndrome?}

According to SCADDING [5], diseases can be defined by four characteristics that often follow in sequence: 1) clinical description (syndrome), 2) disorder of structure (morbid anatomy), 3) disorder of function (pathophysiology) and 4) causation (aetiology). At first glance, the term COPD fulfils them all. First, it is a syndrome, as defined by the presence of some cardinal symptoms (dyspnoea, cough and sputum production) [1]. Second, it has a characteristic morbid pathology, including abnormal remodelling of airways structure (narrowing of the lumen, increase in size of mucosal glands, cellular infiltrates in small and large airways, and actual deposition of peribronchial fibrous tissue), destruction of the parenchyma (emphysema) and compromise of the vessels responsible for blood perfusion [6]. Third, the presence of poorly reversible airflow limitation, as measured by the forced expiratory volume in $1 \mathrm{~s} /$ forced vital capacity ratio, is a core pathophysiological characteristic of the current COPD definition [1]. Finally, epidemiological and clinical studies have shown that COPD has different causative agents, including genetic abnormalities (e.g. $\alpha_{1}$-antitrypsin deficiency), cigarette smoke, exposure to biomass fuels, past infection of the lung, history of asthma and abnormal lung development [1]. In addition, population studies have shown that in a minority, but still sizeable proportion of spirometrically detected cases of COPD, no causative agent can be identified $[7,8]$.

Recent studies, however, question some of these traditional concepts. For instance, some subjects experience symptoms that are similar, if not identical, to those of patients with COPD (including episodes of "exacerbation") but fail to have abnormal spirometry $[9,10]$. Likewise, before the advent of computed tomography (CT), only a careful pathological organ examination was able to detect the presence of emphysema, whereas now CT can identify and quantify in vivo and noninvasively the structural changes of emphysema and/or the presence of airways abnormalities, despite the absence of airflow limitation [11, 12]. It seems, therefore, that the "defining characteristic" of COPD (airflow limitation) does not always accompany the presence of the "characteristic" symptoms and/or morbid pathology of the disease. There exists overwhelming evidence that most cases of COPD in the developed world relate to the smoking of cigarettes. As less developed societies evolve, the smoking habit increases in prevalence, and its importance as a defined and preventable cause of the disease needs to be emphasised. However, the definition of the disease has narrowly been used as synonymous with cigarette smoking, thus creating a degree of confusion that should not exist as many individuals with the syndrome have never smoked $[7,8,13,14]$.

So, is COPD a disease or a syndrome? According to the arguments discussed earlier, COPD clearly is a syndrome, as defined by a set of medical signs and symptoms that are correlated with each other without necessarily tying them to a single identifiable pathogenesis. However, in his very thoughtful discussion about the meaning of disease, syndrome or clinical entity, SCADDING [5] concluded that it really makes no major difference to use either, as long as an agreed term is accepted and shared by the medical community. Thus, given that the term COPD is now finally well known and accepted, and does convey a description of its main clinicophysiological characteristics, we would propose: 1) a minimal change in its name from chronic obstructive pulmonary "disease", where it seems to refer to one single entity, to chronic obstructive pulmonary "diseases" (just adding the "s"), a change that would allow alignment of the accepted terminology while expanding the reality to include the currently known and also the future defined causes of COPD [15], and 2) the following new definition:

COPDs is a clinical syndrome characterised by chronic respiratory symptoms, structural pulmonary abnormalities and/or lung function impairment that results from the interaction of different environmental and genetic factors. 
The type and proportion of respiratory symptoms (breathlessness, cough, sputum production, wheezing and chest tightness), structural pulmonary abnormalities (airway and pulmonary vessels remodelling, emphysema and bronchiectasis) and/or lung function abnormalities (airflow limitation, bronchial hyperresponsiveness, gas trapping and increased lung volumes, low diffusion capacity, and abnormal pulmonary gas exchange) vary greatly both between patients and within the same patient over time, hence making COPDs both heterogeneous and complex [16]. These pulmonary abnormalities are often accompanied by extrapulmonary concomitant diseases (cardiovascular, diabetes, skeletal muscle dysfunction, osteoporosis, lung cancer, depression and anxiety) that also contribute to the clinical presentation, therapeutic needs and prognosis of these patients $[17,18]$. Finally, and importantly, COPDs result from the interaction of several environmental exposures (active and passive smoking, indoor cooking, workplace exposures, ambient pollution, and infections) and genetic factors ( $\alpha_{1}$-antitrypsin deficiency and others still not entirely defined) that, depending on the timing of the interaction(s), can cause abnormal lung development and/or accelerate the decline of lung function that occurs physiologically with ageing.

\section{An improved taxonomy proposal}

Taxonomy is the area of science dedicated to classification $[19,20]$. The current taxonomy of human diseases is still based on the paradigm first proposed by Sir William Osler at the end of the 19th century [21]. The so-called Oslerian paradigm classifies human diseases on the basis of the principal organ system in which symptoms and signs are manifested, and in which gross anatomical pathology and histopathology are correlated [22]. This taxonomy has been extremely useful for clinical practice because it considers a limited number of recognisable patterns of signs and symptoms that may not have a single identifiable pathogenetic paradigm (i.e. as discussed earlier, syndromes) [21]. Yet, it could not have considered the new knowledge about disease aetiology that basic research has produced at the molecular level over the past 50 years. Clearly, it is at this level where drugs find their targets, and where genetic variation and epigenetic regulation contribute to the individual development and progression of disease $[19,20]$. There is, therefore, a need to reform the taxonomy of human diseases in order to move towards personalised and precise medicine [22-24]. COPDs do not escape this debate.

The COPDs taxonomy we propose in the following is mostly based on clinical grounds and uses most of the disease-defining characteristics proposed by SCADDING [5] (figure 1). We readily acknowledge, however, that advances in our understanding of the pathobiological basis of COPD (i.e. endotypes) will eventually facilitate the identification and validation of biomarkers that may modify this classification and could help clinicians deliver a more personalised and precise therapy to different COPD patients [2, 25-27]. Although we provide guidance for therapy, we do not dwell on its details because it is beyond the intent of this review, using it only as support for the taxonomic change proposed here. We think this proposal will allow a more precise definition of the specific nature of a patient's problem (treatable traits [4]), thus providing a better delineation of the population to be included in observational and therapeutic trials, and importantly a more personalised approach to therapy.

\section{Pre-COPD}

Some individuals complain of symptoms similar those of patients with COPDs (they can even "exacerbate") $[9,10,28]$ and/or have CT evidence of abnormal lung structure (e.g. emphysema) $[11,12]$ in

\begin{tabular}{|l|c|c|}
\hline $\begin{array}{l}\text { Scadding } \\
\text { conditions }\end{array}$ & Pre-COPDs & COPDs \\
\hline Function & No airflow limitation & Airflow limitation \\
\hline Symptoms & $\begin{array}{c}\text { Cough; sputum; } \\
\text { dyspnoea }\end{array}$ & Dyspnoea; cough; sputum \\
\hline Structure & $\begin{array}{c}\text { Emphysema; abnormal } \\
\text { airways }\end{array}$ & Emphysema; abnormal airways \\
\hline Aetiology & Genetic (AAT deficiency); cigarettes; biomass; infectious; asthma; developmental; unknown \\
\hline
\end{tabular}

FIGURE 1 Proposed taxonomy of chronic obstructive pulmonary diseases (COPDs) using the principles of ScADDING [5]. Different aetiologies can be responsible for the expression of COPDs with or without airflow limitation. See text for further details. 
the absence of airflow limitation. Strictly speaking, therefore, these individuals do not fulfil the current diagnostic criteria of COPD [1], but, clearly, are not "healthy" subjects and actually manifest abnormal breathing mechanics during increased ventilatory demand [29]. Furthermore, over time, these individuals are at higher risk of developing abnormal airflow limitation, albeit, admittedly, not all of them do [30]. All in all, these observations have led to uncertainties about how to classify these individuals. To address these uncertainties, here we propose to take a page from other areas of medical taxonomy that have used the prefix "pre-" to identify states that are associated with an increased risk (not necessarily the certainty) of developing a disease and classify these individuals as "pre-COPD". The prefix "pre-" is derived from the Latin and means "prior to", "in advance of", "early", "beforehand" or "before" and it has already been used by other medical fields for a very long time to describe a diagnosis such as pre-diabetes [31] or pre-eclampsia [32], to name only a few well-established medical conditions. As is the case for these entities, the full-blown disease may not develop at all, especially if adequate preventive measures are taken, thus highlighting its public health relevance. In fact, this pre-COPD category was already acknowledged by the Global Initiative for Chronic Obstructive Lung Disease (GOLD) Global Strategy for the Diagnosis, Management, and Prevention of Chronic Obstructive Pulmonary Disease in 2006 as GOLD 0 [33], although it was removed in later revisions of the document, mostly because of the realisation that not all GOLD 0 individuals eventually developed airflow limitation [1,34]. Yet, because this concept may open new windows of opportunity for early intervention and prevention, we propose that the term "pre-COPDs" be used for individuals without persistent airflow limitation, but who complain of dyspnoea, cough and/or sputum production and have structural abnormalities (emphysema by CT scan).

\section{Genetic COPD (COPDg)}

The causative relationship between one single gene defect $\left(\alpha_{1}\right.$-antitrypsin) and the development of emphysema was first established by ERIKSSON [35] back in 1965. This particular type of COPD characteristically affects younger individuals, involves primarily the development of emphysema in the lower lobes of the lungs, and may be associated with bronchiectasis, "asthma" and liver involvement. Importantly, it is underdiagnosed and can be treated with specific augmentation therapy, thereby justifying its own subgrouping [36]. Modern research has identified other genetic abnormalities that also increase the risk of developing chronic airflow limitation [37, 38], particularly in young populations [39-42]. A better understanding of the genetic basis of some patients with COPDg should enable the identification and validation of biomarkers that lead 1) to their correct classification and 2) to, eventually, novel targeted therapeutic alternatives $[2,43]$.

\section{Cigarette smoking COPD (COPDC)}

This is the most common and best studied type of all the COPDs. Characteristically, the anatomical lesion is that of varying degree of centrilobular emphysema and involvement of small and large airways [44]. A significant volume of basic and clinical research associates COPDc to an abnormal inflammatory response

\begin{tabular}{|c|c|c|c|c|c|c|c|}
\hline \multicolumn{7}{|c|}{ Generic treatment } \\
\cline { 2 - 7 } & \multicolumn{7}{|c|}{ Maintain a healthy lifestyle: aim for ideal body weight, exercise, appropriate vaccination } \\
If symptomatic use inhaled bronchodilators
\end{tabular}

FIGURE 2 Specific therapeutic and prophylactic interventions to consider in different taxonomic groups of chronic obstructive pulmonary diseases (COPDs). Although separated for the predominant aetiology, some patients may have different mechanisms contributing to the final expression of the disease. AAT: $\alpha_{1}$-antitrypsin; ICS: inhaled corticosteroid. 
to the inhaled products of cigarette combustion [45]. However, most smokers respond to the inhalation of cigarettes with an inflammatory response and not all of them develop COPD, thus providing support for the existence of other mechanisms responsible for the development of COPDc. These mechanisms include: 1) an imbalance between proteases and antiproteases, whereby the former propagate the inflammatory response with destruction of the lung [46], 2) an abnormal immunological reaction that results in some degree of autoimmunity and destruction of elastin and lung parenchyma [47], and, finally, 3) uncontrolled autophagy [48], enhanced apoptosis [49] and/or defective catabasis that results in a process of accelerated ageing (i.e. accelerated decline of lung function with age) [50]. Ideally, these different biological mechanisms (or endotypes) should be identified by appropriately validated biomarkers that can help the clinician decide what would be the more appropriate therapeutic alternative for a given patient (figure 2) [2].

\section{Biomass exposure COPD (COPDb)}

In well-conducted epidemiological studies, $\mathrm{COPDb}$ is the most important cause of COPDs in certain regions of developing countries $[13,51]$. This is particularly relevant for the disease seen in females in these regions, where it can account for the majority of the cases diagnosed [13]. Supporting the concept that this represents a different and specific type of COPD is the fact that detailed studies of these patients show less emphysema and more airways involvement, as well as a more benign decline in lung function over time than patients with COPDc [52-54]. Recent data from studies that have implemented substitution of open flame biomass burning with ventilated cooking furnaces have shown a reduction of COPDb prevalence and symptoms [55]. Finally, we cannot discard the fact that many patients with COPDb live at high altitude (where they use biomass in closed environments as a source of heat) and may develop important cor pulmonale due to the associated hypoxaemia at high altitude [13].

\section{COPD due to infections (COPDi)}

As is the case of COPDb, most clinicians around the globe see nonsmoking subjects with persistent airflow limitation whose only known antecedent is that of previous lung infections. They may be that of repeated events during infancy, classically associated with respiratory syncytial and other viruses [56] or a well-documented history of (treated) tuberculosis, as has been repeatedly observed in certain parts of the world [13, 57]. The interaction between infectious processes and the resulting limitation to airflow is poorly defined, but it is likely due to abnormal repair processes that lead to fibrosis and distortion of the airways. There are very few studies that have addressed the therapeutic implications of COPDi. Most of what we know relates to the effect of antituberculous drugs on the disease itself rather than on its effect in modulating the repair process in the case of tuberculosis or the benefits that may accrue from the treatment of the infectious components of bronchiectasis when they are present [58]. Recent research, however, has clearly shown that the presence of bronchiectasis in patients with COPDs conveys a poor prognosis [59], supporting the need to address this type of COPD with more emphasis on the reasons for the progression of the disease.

\section{Asthma and COPD (COPDa)}

Whether asthma is a necessary component in the genesis of COPD has been a debatable issue for a long time $[60,61]$, but it is recognised that patients with severe, uncontrolled asthma can develop chronic airflow limitation without any of the other risk factors and, as such, represent a specific group of patients with COPD (COPDa) [62]. In addition to the presence of symptoms and poorly reversible obstruction, detailed physiological and pathological studies have documented the presence of varying degrees of loss of lung elastic recoil and emphysema in patients with COPDa $[63,64]$. This asthma-COPD overlap has been the subject of intense recent interest $[27,65]$, but further studies need to be conducted in populations with COPDa because the advent of biologicals that are effective in selected cases of severe asthma may benefit these individuals [66].

\section{COPD due to abnormal lung development (COPDd)}

Lungs are not fully developed at birth [67]; they continue to grow and mature, reaching a peak at 16-18 years in females and 20-25 years in males [68]. Many environmental (passive smoking, repeated infections and poor nutrition, among others) and genetic factors (most still unknown) can alter lung growth in utero, during infancy and during adolescence [69]. Recent research has shown that among adult patients fulfilling the diagnostic criteria of COPD [1], about half had a lung function trajectory through life that started from a normal peak lung function value in early adulthood followed by an enhanced rate of decline with age (i.e. they follow the traditional FLETCHER and PETO [70] model), whereas the other half started the journey from a lower peak lung function value in early adulthood and could be diagnosed with COPD without an enhanced rate of lung function decline [14]. It is entirely conceivable that these two trajectories identify different types of COPD (COPDs versus COPDd) that have different pathobiology (endotypes), prognosis and treatment requirements. We need to identify and validate biomarkers of these 
two forms of COPDs to develop novel therapies and/or repurpose existing therapies [43]. It is possible that cases attributed to asthma during childhood (COPDa) may relate to the fact that a child with lung developmental problems is likely to experience some form of respiratory symptoms (e.g. dyspnoea on exercise and wheezing) and be diagnosed with "asthma" if taken to a paediatrician [71].

\section{COPD of unknown cause (COPDu)}

Well-conducted studies determining the prevalence of COPD have observed that a significant proportion of subjects with airflow limitation provide no associated factors that can be invoked as the cause of the abnormality and therefore would qualify as idiopathic (i.e. of unknown cause) [8]. This type of COPD remains poorly studied, but seems to have a better prognosis than those types mentioned earlier. The majority of the patients manifest a mild to moderate degree of airflow limitation and this tends to remain so over time [28]. This type of COPD may be the end result of several different factors capable of influencing its clinical expression, such as sex, occupational exposure, education, income as well as other as-yet undetermined epigenetic phenomena [7]. Likewise, it may represent the individuals who have a limitation in the growth phase of their lungs and develop COPD at a normal rate of the natural decline of lung function (COPDd), as discussed earlier, since only a minority of physicians ask about early-life events when seeing a COPD patient [72]. Given that we do not know the causative agent(s), thus negating the possibility of primary or secondary prevention, there is a need for studies specifically designed to address COPDu.

\section{Conclusions}

The concepts surrounding COPD have changed significantly from a quasi-orphan, unpreventable and untreatable disease when first described by Rene Laennec in 1821 [73] to one where research has opened windows to its pathogenesis, causative agents, clinical expressions and therapy. Supported by the knowledge acquired over decades, this review proposes to keep the now well-known and accepted term of COPD but, at the same time, recognises that it is an umbrella term that describes a clinical syndrome that includes several COPDs (figure 1) which, importantly, may eventually benefit from different preventive and therapeutic strategies (figure 2). We acknowledge that subjects may have multiple exposures, thereby making a specific taxonomic classification difficult. However, this is true for every known disease, and the grouping of patients into their most likely aetiological cause will facilitate studies designed to test therapeutic programmes and help clinicians understand the natural course and approaches to individual patients. We also recognise that this taxonomic proposal has to be specifically applied to the planning of studies aimed at better defining the pathobiological mechanisms involved in the genesis of the different groups and their therapy. For instance, we do not know if different COPDs may be associated with specific clinical presentations (phenotypes), such as the presence of chronic bronchitis, emphysema, exacerbations and/or comorbidities, to name only a few. Similarly, most of what we know today about COPD treatment is derived from studies centred almost exclusively on COPDc, yet these results have been extrapolated directly to other causes of COPD in the many guidelines that have been developed around the world. Some of these COPDs may be amenable to effective primary and secondary prevention [71], so we should all be hopeful that by applying them, we can decrease the important burden that this disease imposes on society. Finally, this taxonomic proposal is not definitive and, as stated, it is only a proposal. We expect to stimulate discussion and, if adopted, changes may be added as our knowledge about COPDs increases.

\section{References}

1 Global Initiative for Chronic Obstructive Lung Disease. Global Strategy for the Diagnosis, Management, and Prevention of Chronic Obstructive Pulmonary Disease. 2017. http://goldcopd.org/gold-2017-global-strategy-diagnosismanagement-prevention-copd/ Date last accessed: November 16, 2016.

2 Woodruff PG, Agusti A, Roche N, et al. Current concepts in targeting chronic obstructive pulmonary disease pharmacotherapy: making progress towards personalised management. Lancet 2015; 385: 1789-1798.

3 Chu JH, Hersh CP, Castaldi PJ, et al. Analyzing networks of phenotypes in complex diseases: methodology and applications in COPD. BMC Syst Biol 2014; 8: 78

4 Agusti A, Bel E, Thomas M, et al. Treatable traits: toward precision medicine of airway diseases. Eur Respir J 2016; 47: 410-419.

$5 \quad$ Scadding JG. Health and disease: what can medicine do for philosophy? J Med Ethics 1988; 14: 118-124.

6 Hogg JC. Pathophysiology of airflow limitation in chronic obstructive pulmonary disease. Lancet 2004; 364: 709-721.

7 Lamprecht B, McBurnie MA, Vollmer WM, et al. COPD in never smokers: results from the population-based burden of obstructive lung disease study. Chest 2011; 139: 752-763.

8 Celli BR, Halbert RJ, Nordyke RJ, et al. Airway obstruction in never smokers: results from the Third National Health and Nutrition Examination Survey. Am J Med 2005; 118: 1364-1372.

9 Woodruff PG, Barr RG, Bleecker E, et al. Clinical significance of symptoms in smokers with preserved pulmonary function. N Engl J Med 2016; 374: 1811-1821.

10 Rodriguez-Roisin R, Han MK, Vestbo J, et al. Chronic respiratory symptoms with normal spirometry. a reliable clinical entity? Am J Respir Crit Care Med 2017; 195: 17-22. 
11 Wilson DO, Weissfeld JL, Balkan A, et al. Association of radiographic emphysema and airflow obstruction with lung cancer. Am J Respir Crit Care Med 2008; 178: 738-744.

12 Alcaide AB, Sanchez-Salcedo P, Bastarrika G, et al. Clinical features of smokers with radiological emphysema but without airway limitation. Chest 2017; 151: 358-365.

13 Salvi SS, Barnes PJ. Chronic obstructive pulmonary disease in non-smokers. Lancet 2009; 374: 733-743.

14 Lange P, Celli B, Agustí A, et al. Lung-function trajectories leading to chronic obstructive pulmonary disease. $N$ Engl J Med 2015; 373: 111-122.

15 Rennard SI, Vestbo J. The many "small COPDs": COPD should be an orphan disease. Chest 2008; $134: 623-627$.

16 Agusti A. The path to personalized medicine in COPD. Thorax 2014; 69: 857-864.

17 Divo M, Cote C, de Torres JP, et al. Comorbidities and risk of mortality in patients with chronic obstructive pulmonary disease. Am J Respir Crit Care Med 2012; 186: 155-161.

18 Divo MJ, Casanova C, Marin JM, et al. COPD comorbidities network. Eur Respir J 2015; 46: 640-650.

19 Hofmann-Apitius M, Alarcón-Riquelme ME, Chamberlain C, et al. Towards the taxonomy of human disease. Nat Rev Drug Discov 2015; 14: 75-76.

20 Kola I, Bell J. A call to reform the taxonomy of human disease. Nat Rev Drug Discov 2011; 10: 641-642.

21 Vanfleteren LEGW, Kocks JWH, Stone IS, et al. Moving from the Oslerian paradigm to the post-genomic era: are asthma and COPD outdated terms? Thorax 2014; 69: 72-79.

22 Loscalzo J, Barabasi AL. Systems biology and the future of medicine. Wiley Interdiscip Rev Syst Biol Med 2011; 3 : 619-627.

23 Collins FS, Varmus H. A new initiative on precision medicine. N Engl J Med 2015; 372: 793-795.

24 Jameson JL, Longo DL. Precision medicine - personalized, problematic, and promising. N Engl J Med 2015; 372: 2229-2234.

25 Kostikas K, Bakakos P, Papiris S, et al. Systemic biomarkers in the evaluation and management of COPD patients: are we getting closer to clinical application? Curr Drug Targets 2013; 14: 177-191.

26 Agusti A, Celli B, Faner R. What does endotyping mean for treatment in chronic obstructive pulmonary disease? Lancet 2017; 390: 980-987.

27 Pavord ID, Beasley R, Agusti A, et al. After asthma: redefining airways diseases. Lancet 2018; 391: 350-400.

28 Labonté LE, Tan WC, Li PZ, et al. Undiagnosed chronic obstructive pulmonary disease contributes to the burden of health care use. Data from the CanCOLD study. Am J Respir Crit Care Med 2016; 194: 285-298.

29 Ofir D, Laveneziana P, Webb KA, et al. Mechanisms of dyspnea during cycle exercise in symptomatic patients with GOLD stage I chronic obstructive pulmonary disease. Am J Respir Crit Care Med 2008; 177: 622-629.

30 Vestbo J, Lange P. Can GOLD Stage 0 provide information of prognostic value in chronic obstructive pulmonary disease? Am J Respir Crit Care Med 2002; 166: 329-332.

31 Barach JH. The prediabetic state and its clinical importance. J Fla Med Assoc 1950; 37: 145-148.

32 Bonney V. "Pre-eclampsia" at the twenty-fourth week; acute toxaemia; caesarean section. Proc R Soc Med 1914; 7: $118-121$.

33 Rabe KF, Hurd S, Anzueto A, et al. Global strategy for the diagnosis, management, and prevention of chronic obstructive pulmonary disease: GOLD executive summary. Am J Respir Crit Care Med 2007; 176: 532-555.

34 Vestbo J, Hurd SS, Agustí A, et al. Global strategy for the diagnosis, management and prevention of chronic obstructive pulmonary disease: GOLD executive summary. Am J Respir Crit Care Med 2013; 187: 347-365.

35 Eriksson S. Studies in alpha 1-antitrypsin deficiency. Acta Med Scand Suppl 1965; 432: 1-85.

36 Chapman KR, Burdon JGW, Piitulainen E, et al. Intravenous augmentation treatment and lung density in severe $\alpha_{1}$ antitrypsin deficiency (RAPID): a randomised, double-blind, placebo-controlled trial. Lancet 2015; 386: 360-368.

37 Hobbs BD, de Jong K, Lamontagne M, et al. Genetic loci associated with chronic obstructive pulmonary disease overlap with loci for lung function and pulmonary fibrosis. Nat Genet 2017; 49: 426-432.

38 Wain LV, Shrine N, Artigas MS, et al. Genome-wide association analyses for lung function and chronic obstructive pulmonary disease identify new loci and potential druggable targets. Nat Genet 2017; 49: 416-425.

39 Qiao D, Lange C, Beaty TH, et al. Exome sequencing analysis in severe, early-onset chronic obstructive pulmonary disease. Am J Respir Crit Care Med 2016; 193: 1353-1363.

40 Foreman MG, Kong X, DeMeo DL, et al. Polymorphisms in surfactant protein-D are associated with chronic obstructive pulmonary disease. Am J Respir Cell Mol Biol 2011; 44: 316-322.

41 Foreman MG, Zhang L, Murphy J, et al. Early-onset chronic obstructive pulmonary disease is associated with female sex, maternal factors, and African American race in the COPDGene Study. Am J Respir Crit Care Med 2011; 184: 414-420.

42 DeMeo DL, Mariani TJ, Lange C, et al. The SERPINE2 gene is associated with chronic obstructive pulmonary disease. Am J Hum Genet 2006; 78: 253-264.

43 Holgate S, Agusti A, Strieter RM, et al. Drug development for airway diseases: looking forward. Nat Rev Drug Discov 2015; 14: 367-368.

44 Hogg JC, Macklem PT, Thurlbeck WM. Site and nature of airway obstruction in chronic obstructive lung disease. N Engl J Med 1968; 278: 1355-1360.

45 Hogg JC, Chu F, Utokaparch S, et al. The nature of small-airway obstruction in chronic obstructive pulmonary disease. N Engl J Med 2004; 350: 2645-2653.

46 Barnes PJ, Shapiro SD, Pauwels RA. Chronic obstructive pulmonary disease: molecular and cellular mechanisms. Eur Respir I 2003; 22: 672-688.

47 Cosio M, Saetta M, Agusti A. Immunological aspects of COPD. N Engl J Med 2009; 360: 2445-2454.

48 Choi AMK, Ryter SW, Levine B. Autophagy in human health and disease. N Engl J Med 2013; 368: 651-662.

49 Kasahara Y, Tuder RM, Cool CD, et al. Expression of 15-lipoxygenase and evidence for apoptosis in the lungs from patients with COPD. Chest 2000; 117: 5 Suppl. 1, 260S.

50 Mercado N, Ito K, Barnes PJ. Accelerated ageing of the lung in COPD: new concepts. Thorax 2015; 70: 482-489.

51 Diaz E, Bruce N, Pope D, et al. Lung function and symptoms among indigenous Mayan women exposed to high levels of indoor air pollution. Int J Tuberc Lung Dis 2007; 11: 1372-1379.

52 Ramírez-Venegas A, Sansores RH, Pérez-Padilla R, et al. Survival of patients with chronic obstructive pulmonary disease due to biomass smoke and tobacco. Am J Respir Crit Care Med 2006; 173: 393-397. 
Liu S, Zhou Y, Wang X, et al. Biomass fuels are the probable risk factor for chronic obstructive pulmonary disease in rural South China. Thorax 2007; 62: 889-897.

Hu G, Zhou Y, Tian J, et al. Risk of COPD from exposure to biomass smoke: a metaanalysis. Chest 2010; 138 $20-31$.

Zhou Y, Zou Y, Li X, et al. Lung function and incidence of chronic obstructive pulmonary disease after improved cooking fuels and kitchen ventilation: a 9-year prospective cohort study. PLoS Med 2014; 11: e1001621.

Berry CE, Billheimer D, Jenkins IC, et al. A distinct low lung function trajectory from childhood to the fourth decade of life. Am J Respir Crit Care Med 2016; 194: 607-612.

Menezes AM, Hallal PC, Perez-Padilla R, et al. Tuberculosis and airflow obstruction: evidence from the PLATINO study in Latin America. Eur Respir J 2007; 30: 1180-1185.

Hnin K, Nguyen C, Carson KV, et al. Prolonged antibiotics for non-cystic fibrosis bronchiectasis in children and adults. Cochrane Database Syst Rev 2015; 8: CD001392.

Martinez-Garcia MA, de la Rosa D, Soler-Cataluna JJ, et al. Prognostic value of bronchiectasis in patients with moderate-to-severe chronic obstructive pulmonary disease. Am J Respir Crit Care Med 2013; 187: 823-831.

Orie NG, Slutter HJ, De Vries K, et al. [Chronic nonspecific respiratory diseases]. Ned Tijdschr Geneeskd 1961; 105: 2136-2139.

Sluiter HJ, Koëter GH, de Monchy JG, et al. The Dutch hypothesis (chronic non-specific lung disease) revisited. Eur Respir J 1991; 4: 479-489.

McGeachie MJ, Yates KP, Zhou X, et al. Genetics and genomics of longitudinal lung function patterns in individuals with asthma. Am J Respir Crit Care Med 2016; 194: 1465-1474.

Gelb AF, Zamel N. Unsuspected pseudophysiologic emphysema in chronic persistent asthma. Am J Respir Crit Care Med 2000; 162: 1778-1782.

Senhorini A, Ferreira DS, Shiang C, et al. Airway dimensions in fatal asthma and fatal COPD: overlap in older patients. COPD 2013; 10: 348-356.

Bateman ED, Reddel HK, van Zyl-Smit RN, et al. The asthma-COPD overlap syndrome: towards a revised taxonomy of chronic airways diseases? Lancet Respir Med 2015; 3: 719-728.

Busse WW. Biologicals for asthma in patients with asthma-COPD overlap. Lancet Respir Med 2017; 5: 175-177. Bush A. Lung development and aging. Ann Am Thorac Soc 2016; 13: Suppl. 5, S438-S446.

Kohansal R, Martinez-Camblor P, Agustí A, et al. The natural history of chronic airflow obstruction revisited: an analysis of the Framingham offspring cohort. Am J Respir Crit Care Med 2009; 180: 3-10.

Martinez FD. Early-life origins of chronic obstructive pulmonary disease. N Engl J Med 2016 375: 871-878.

Fletcher C, Peto R. The natural history of chronic airflow obstruction. Br Med J 1977; 1: 1645-1648.

Agustí A, Celli B. Natural history of COPD: gaps and opportunities. ERJ Open Res 2017; 3: 00117-2017.

Bolton CE, Bush A, Hurst JR, et al. Are early life factors considered when managing respiratory disease? A British Thoracic Society survey of current practice. Thorax 2012;67: 1110

3 Snider GL. Emphysema: the first two centuries - and beyond. A historical overview, with suggestions for future research: part 1. Am Rev Respir Dis 1992; 146: 1334-1344. 